\title{
ISOTHERMAL DENDRITIC GROWIH - A LOW GRAVITY EXPERIMENT
}

M. E. Glicksman, R. C. Hahn, T. A. Lograsso, E. R. Rubinstein and M. E. Selleck

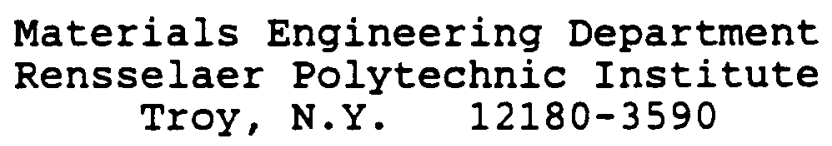

and

E. Winsa

NASA Lewis Research Center

Cleveland, Ohio 44135

Submitted to NASA Headquarters Code $E-N$ as a publication as a part of the Elight Experiment Science Review, March 9, 1987. Lewis Research Center, Cleveland, Ohio 44135 


\section{ABSTRACT}

The growth of dendrites in pure melts and alloys is controlled by diffusion-limited transport of heat and/or solute. The presence of temperature or concentration gradients within a molten phase subject to gravitational forces generally promotes convection, which in turn, modifies the diffusion processes. In the case of dendritic growth, where convective flows are threedimensional and complex, the normally isotropic diffusional transport mechanisms are rendered anisotropic. The introduction of anisotropic transport mechanisms alter the fundamental crystal growth behavior. Ground based studies of a pure material, succinonitrile, have shown that kinetic and morphological characteristics of dendritic growth can be strongly affected by convective flows. Experimentally measured growth velocities and dendrite tip radii at undercoolings greater than $1.0 \mathrm{~K}$ agree well with values predicted by theoretical models of dendritic growth based on diffusional transport mechanisms. At undercoolings less than $1.0 \mathrm{~K}$, the experimental data deviates from the predicted values and is the result of convective flows disturbing the otherwise spatialiy isotropic diffusion field. The vigor of melt convection is controlled by several parameters often expressed as a Iumped dimensionless group, the Grashof number $\mathrm{Gr}=\mathrm{g} \beta \Delta \mathrm{T} \ell^{3} / v^{2}$, where $g$ is the acceleration due to gravity; $\beta$ is the volumetric expansion coefficient; $\Delta T$ is the undercooling; $v$ is the kinematic viscosity; and $l$ is the relevant length scale, e.g., the characteristic diffusion distance.

Dendritic growth, by its nature, does not permit independent manipulation of the controlling length scale, $\ell$, which is determined by materials properties (e.g. diffusion coefficient or thermal diffusivity) and the undercooling or supersaturation. The reduction of $g$ through orbital free fall is often the only practical way to lower Gr sufficiently to permit careful observation of the morphological and kinetic characteristics of isothermal dendritic growth. Estimates have been made as to how low gravitational accelerations would have to be reduced in order to observe "convection free" dendritic growth at undercoolings from 0.1 to $1.0 \mathrm{~K}$. The purpose of the proposed microgravity isothermal dendritic growth experiment (IDGE) is to test dendritic growth theory at low undercoolings where growth kinetics are normally overwhelmed at $1 \mathrm{~g}$. A microgravity experiment would allow convection to be reduced to a level which would permit observations in the range of undercoolings 0.1 to $1.0 \mathrm{~K}$ where both tip radius and growth velocity can be measured with high accuracy and then compared with values predicted by transpcrt/stability theory. Previously conducted ground-based studies and the current approach to performing these studies in low earth orbit will be described. 


\section{INIRODUCTION}

Dendritic growth is the most common form of crystal growth in metallic alloys and other systems forming centrosymmetric, simple crystal structures. Dendrites are complex branched forms that grow in specific crystallographic directions and evolve into elaborate 3 dimensional structures. The characteristic branching, see Figure 1, establishes the typical length scales for the microsegregation of chemical species rejected at the growing solid-liquid interfaces. The patterns of chemical segregation have a profound influence on the electrical, mechanical, and corrosion characteristics of the cast material.

Dendritic crystal growth occurs in metals and alloys in the presence of either constitutional supercooling in the case of alloys or thermal supercooling in the case of pure materials. Latent heat or solute is released as the cendrite advances, which changes the local density of the surrounding liquid. Gravitational forces, if present, cause complex, three-dimensional convective flows. These convective flows alter the thermal fields governing dendritic growth, affecting both the growth rate and tip radius of the dendrite [1]. Figure 2 shows the effect of various growth orientations with respect to the gravity

\section{ORIGINAL PAGE IS OF POOR QUALITY}




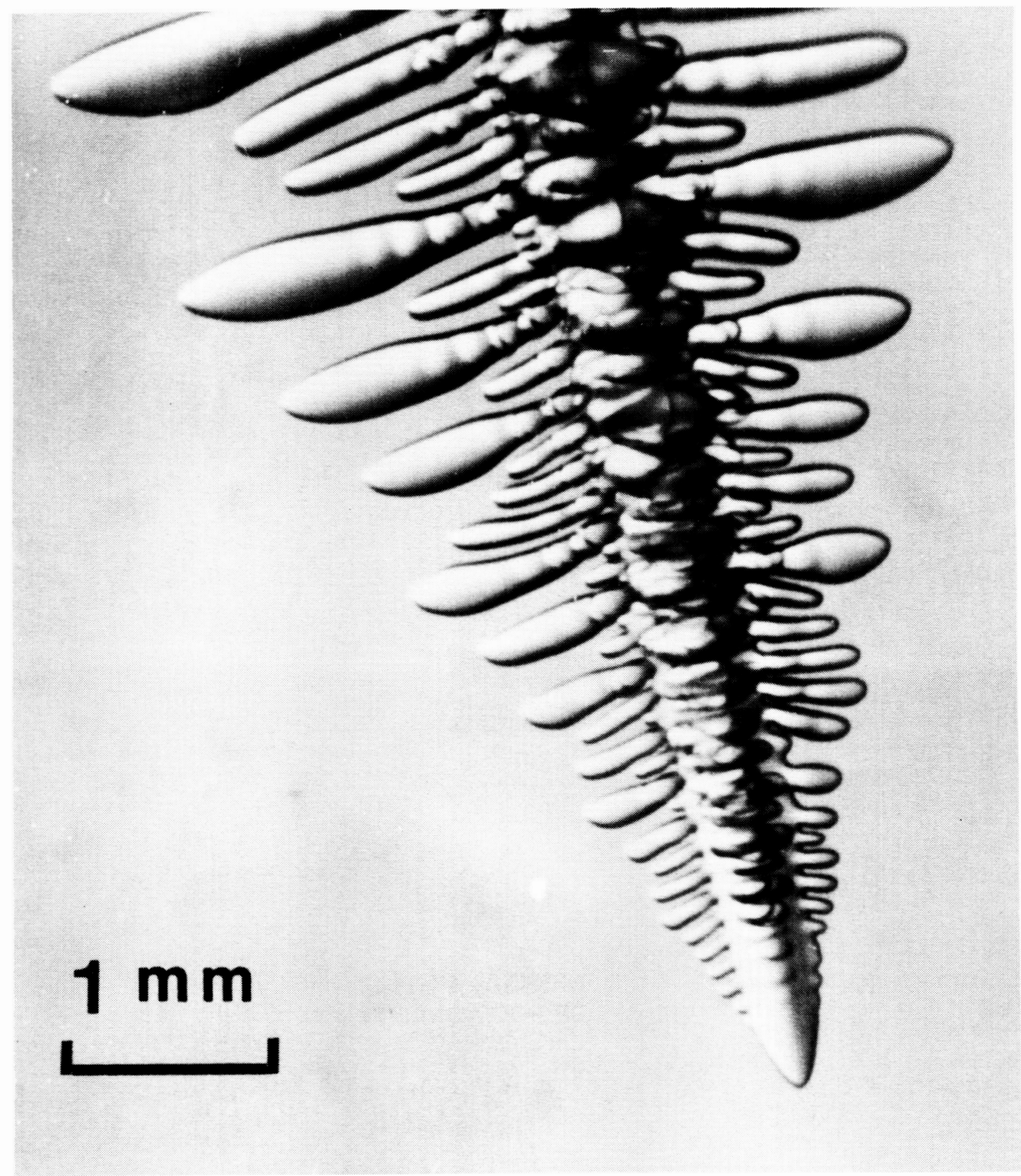

Figure 1 Dendritic crystal growing in succinonitrile. 

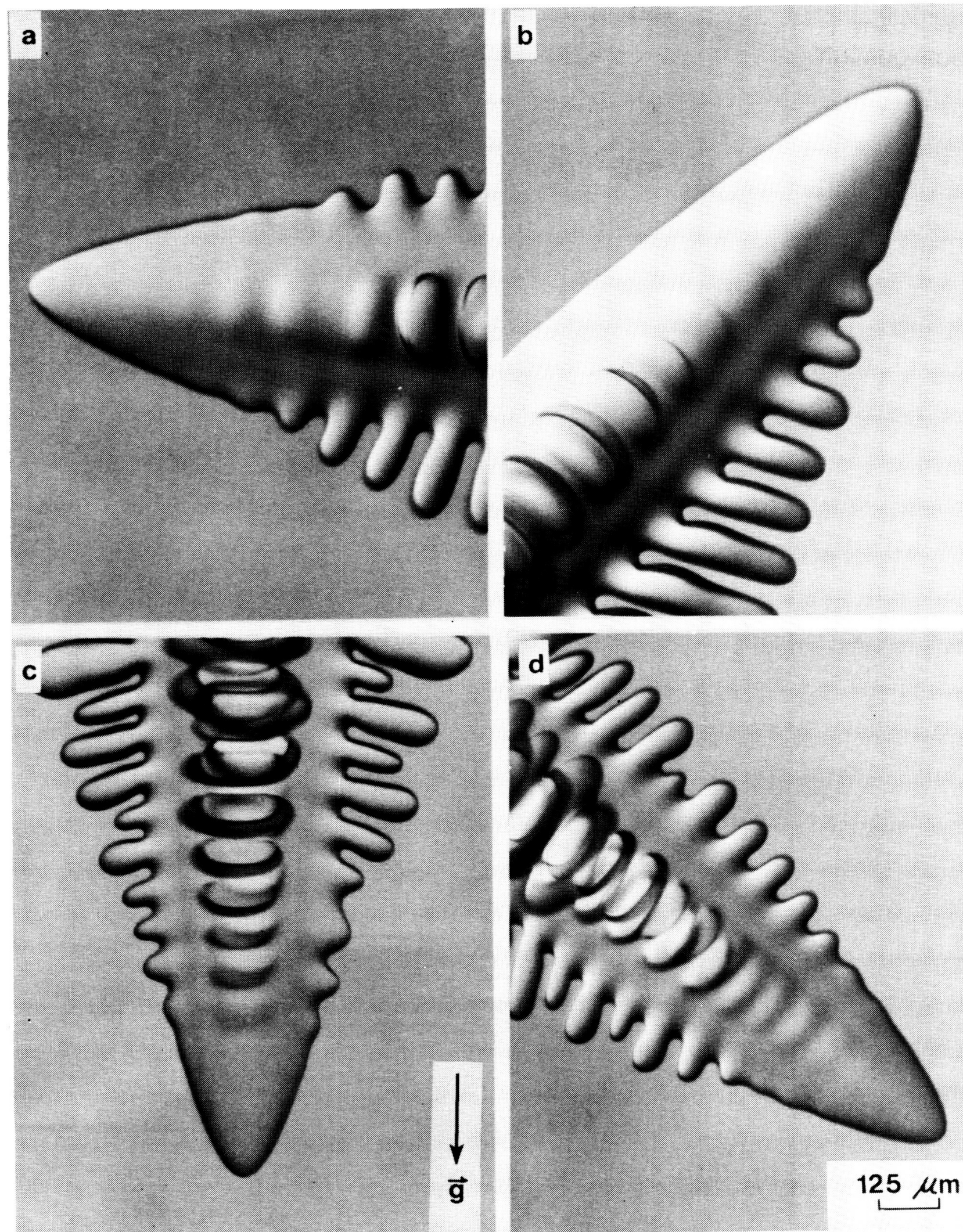

Figure 2 Succinonitrile dendrites show in true growth orientation with respect to gravity: (a) perpendicular to gravity, side branching is suppressed on top; (b) as growth angle is increased side branch is further suppressed on top; (c) parallel to gravity side branches are symmetrical; (d) a slight increase in growth angle results in little noticeable asymmetry. 
ORIGINAL PAGE IS

OF POOR QUALITY

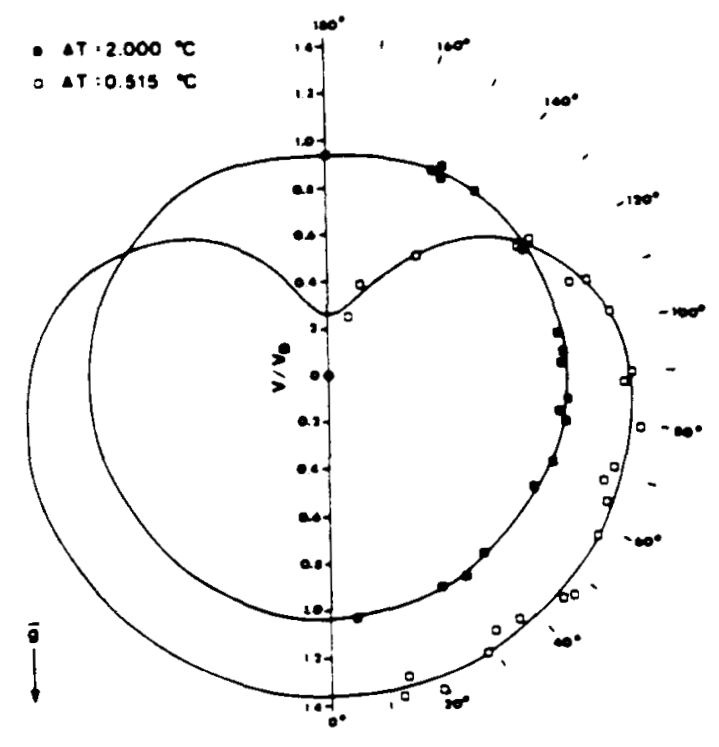

Figure 3 Growth velocity as a function of growth angle with respect to gravity at $2.0 \mathrm{~K}$ and $0.515 \mathrm{~K}$ undercooling.

vector at $1 \mathrm{~g}$ on the morphology and the sidebranch structure of a dendrite. A corresponding spatial anisotropy in the growth rate is shown in Figure 3 . Convective effects have their greatest influence at small undercoolings and, therefore, it would be desirable to quantify the diffusion-limited kinetics of dendritic growth at these undercoolings in the absence of convection, in order to comprehend the influence of convection on the dendritic growth process. Small undercoolings are also advantageous from an experimental point of view since the growth velocities are low and tip radii are large, allowing accurate measurements of both. Determination of both of these quantities is necessary to test dendritic growth theory adequately. It is the intent of the isothermal dendritic growth experiment (IDGE) to test dendritic growth theory in low earth orbit where convection will be reduced to a level that will permit observations of diffusion controlled growth in the range of undercoolings from $0.1 \mathrm{~K}$ to $1.0 \mathrm{~K}$. 


\section{DENDRITIC GROFTH THEORY}

Over the past 30 years, numerous theories have been developed describing steady-state dendritic growth $[2,3]$. Experimental evidence [3] has shown that these theories correctly describe one or more aspects of dendritic growth but none were capable of a complete description of the growth kinetics. The comparison of experimental data to the various models indicate that two of these theories, the Ivantsov model [4] and the Langer and Muller-Krumbhaar stability criterion $[5,6,7]$ when used in combination, adequately predict the kinetics of dendritic growth.

Ivantsov modeled steady-state dendritic growth using heat transport theory, by assuming that a paraboloid of revolution described the shape of the dendrite devoid of sidebranches. Ivantsov also neglected any capillarity effects and molecular attachment kinetics. The solution to the diffusion equation yields an expression for the dimensionless undercooling of the form:

$$
\Delta \Theta=P e \exp (\mathrm{Pe}) \mathrm{E}_{1}(\mathrm{Pe}),
$$

where $\Delta \theta=\left(T_{m}-T_{\infty}\right) /\left(L / C_{p}\right)$ is the dimensionless undercooling; $\mathrm{T}_{\mathrm{m}}$ is the melting temperature; $\mathrm{T}_{\infty}$ is the temperature of the melt far from the interface; $L$ is the latent heat of fusion; $C_{p}$ is the heat capacity; $P e=V R / 2 a$ is the Peclet number; $V$ is the growth velocity; $R$ is the dendrite tip radius; $a$ is the thermal diffusivity and $E_{1}$ is the first exponential integral. This solution, however, does not yield a unique value of growth velocity and tip radius at each undercooling, but provides a set of values of the form $V R=$ constant. A morphological

stability criterion is invoked to obtain the steady-state operating tip radius and growth rate at a given undercooling.

Langer and Muller-Krumbhaar ( $L M-K$ ) have applied linear stability theory to a paraboloidal solid-liquid interface and derived an expression for the stability of a dendritic surface which is marginally stable at the tip and unstable at all other points. A stability criterion was formulated, in the limit of vanishing surface tension, and can be expressed as:

$$
\sigma^{\star}=2 a d_{0} / V^{2}=0.025 \text {, }
$$

where $d_{0}=T_{m} Y C_{p} / L$ is the capillary length, and $Y$ is the solid-liquid sufface energy. The LM-K criterion predicts an independent continuum of operating tip radii and growth rates which can then be combined with the Ivantsov model, $\mathrm{VR}=2 \mathrm{aPe}$. These two equations can be solved yielding a unique growth velocity and tip radius at each undercooling, simultaneously satisfying stability and energy transport. Experimental data for succinonitrile $[1,2]$ agree well with the predicted growth velocities at undercoolings greater than approximately $1.2 \mathrm{~K}$, as shown in Figure 4. At smaller undercoolings, convective flows 


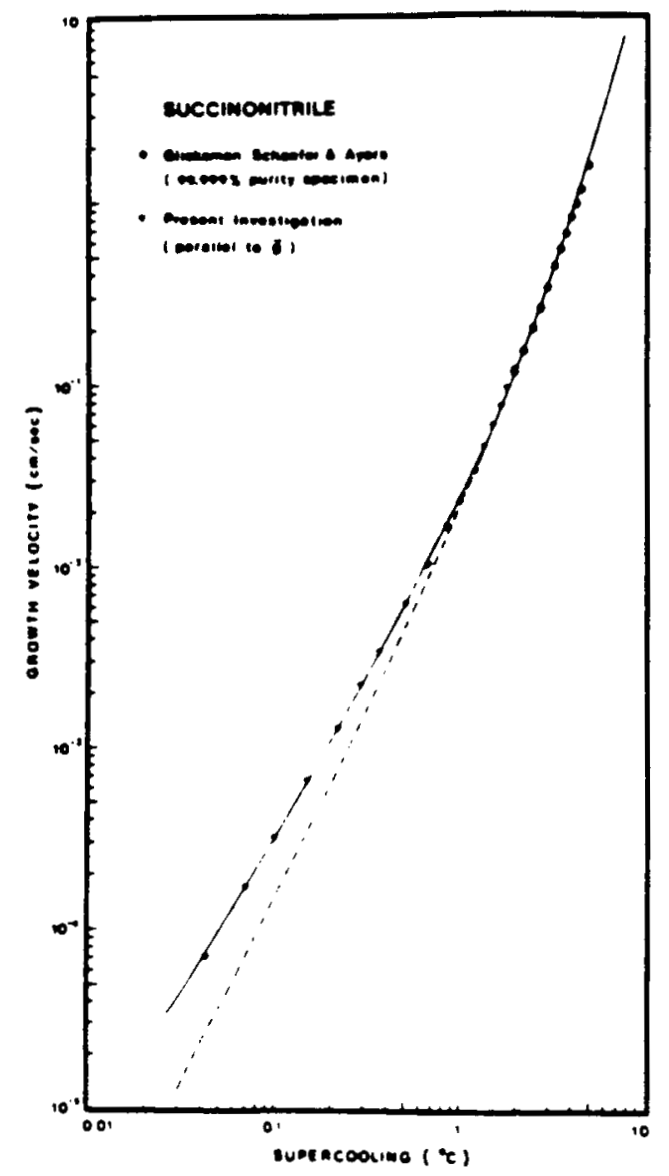

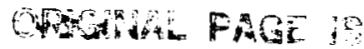

TI OOR QUALITY

Figure 4 Growth

velocity as a

function of undercooling. Calculated values are shown as a dashed line.

begin to disrupt the spatially isotropic diffusive transport of heat and both growth velocity and tip radii begin to deviate appreciably from those predicted from theory [2]. A microgravity dendritic growth experiment is planned in an effort to extend the range of agreement between theory and experiment to lower undercoolings where, at 1g, the growth kinetics are overwhelmed by convection.

A new "mechanism" for the velocity selection in dendritic growth has been advanced in recent years [8-11]. This mechanism has been called "microscopic solvability" and has been applied to dendritic growth and viscous fingering. This new approach is based on the premise that a finite surface tension and crystalline anisotropy introduces a solvability condition, not considered in the LM-K theory, which determines the operating growth velocity. The incorporation of finite surface tension and anisotropy into the equations which govern dendritic growth yields a discrete set of solutions for small undercoolings, each having a unique velocity. The final operating state of the dendrite is then determined by stability analysis around a chosen steadystate shape such that the solution with the largest velocity is the most stable. For small undercoolings, this analysis also yields an explicit relationship between the growth velocity and the degree of crystalline 
anisotropy, $a$, of the form [12]:

$$
v \approx \alpha^{7 / 4}
$$

In addition, the model is able to predict stable sidebranch formation when finite thermal noise about the steady-state solution is introduced. At present, it is unclear whether this model is consistent with available experimental data. Since the analysis has been carried out for small undercoolings, it has been suggested [12] that a check on the validity of this analysis must be performed in a "convection free" environment. The results from the proposed microgravity experiment may assist in the evaluation of this analysis.

\section{ESTIMATE OF REQUIRED MICROGRAVITY LEVEIS}

The reduced microgravity environment in the low earth orbit flown by the space Transportation System (STS) can still give rise to convection which could adversely affect dendritic growth. The measured gravitational environment can be divided into two regimes, a low frequency range (DC to $\left.10^{-3} \mathrm{~Hz}\right)$ and a high frequency range $\left(10^{-3}\right.$ to $\left.10 \mathrm{~Hz}\right)$. The DC component [13] arises from orbital friction and drag forces and is typically $10^{-8} \mathrm{~g}$ to $10^{-4} \mathrm{~g}$ in magnitude. AC accelerations arise from a variety of sources, both intrinsic to the orbit and within the STS orbjter itself, which range in amplitude from $10^{-6} \mathrm{~g}$ to $10^{-1} \mathrm{~g}[13]$. Prior to conducting a microgravity experiment such as the IDGE, it was necessary to determine if the typical gravitational spectrum encountered will affect dendritic growth. An order-of-magnitude estimate of the upper limit of allowable gravitational accelerations can be calculated using a scaling analysis developed by Langbein [14]. According to the analysis, the allowable acceleration level can be estimated as:

$$
a_{0}=\frac{\rho \delta T}{\Delta \rho \nabla T_{0}}\left[\omega^{2}+\left(\nu / L^{2}\right)^{2}\right]^{1 / 2}\left[\omega^{2}+\left(\alpha / L^{2}\right)^{2}\right]^{1 / 2}
$$

where is the density, $\Delta \rho=\beta \Delta T$ the density change induced in the melt with temperature; $\nabla \mathrm{T}_{0}$, in this case, is the undercooling; $6 \mathrm{~T}$ is the allowable temperature deviation of the interface due to convection; $\omega$ is the circular frequency of the acceleration; $v$ is the kinematic viscosity; and $L$ is the length scale over which convection occurs. It is possible to calculate the frequency and amplitude of acceleration required to induce convection sufficient to modify the growth kinetics by substituting appropriate values into the analysis and by assuming that 18 change in temperature, $6 \mathrm{~T}$, will not affect the measured growth parameters within experimental error. The results of these calculations are shown in Figure 5 as a function of undercooling. At high undercoolings, the growth kinetics are relatively insensitive to convection whereas 


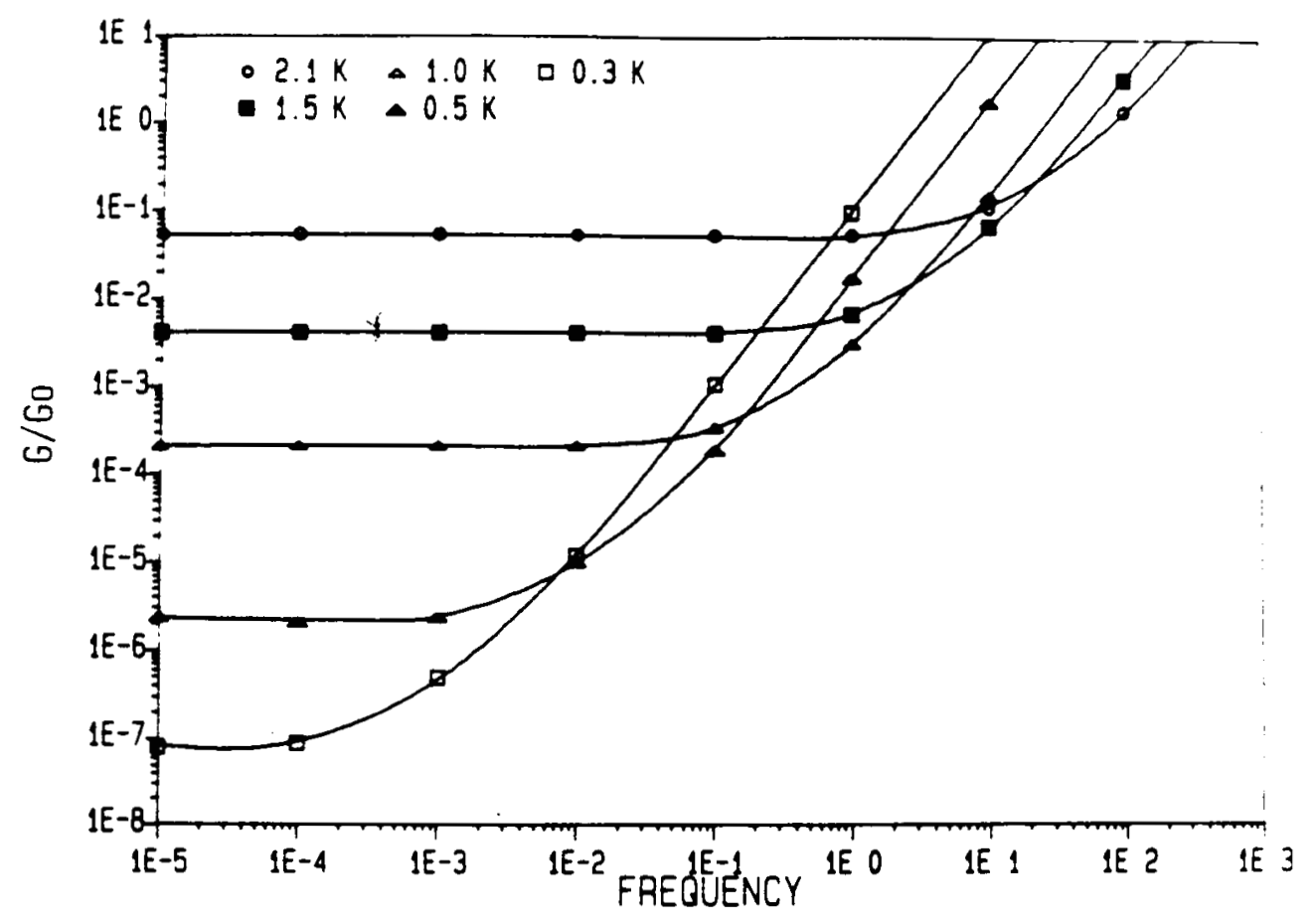

Figure 5 Acceleration limit for the onset of convection at various undercoolings. Any combination of frequency and "G" levels below the calculated curves are sufficient to induce convection which measurably modifies growth kinetics.

at low undercoolings the opposite is true. Furthermore, as the frequency increases, the level of allowable accelerations increases rapidly, indicating that dendritic growth is insensitive to gravitational fluctuations of short duration. It can therefore be assumed that the "typical" STS gravitational environment will probably provide an adequate low-gravity spectrum to study nearly convection-free dendritic growth.

\section{EXPERIMIENT PROFILE}

The flight experiment planned will be divided into two phases , 1) "warmup" and 2) data acquisition cycles. The "warmup" phase consists of raising the temperature of the thermostat from approximately $30^{\circ} \mathrm{C}$ to the operating range of $58^{\circ} \mathrm{C}$ to $62^{\circ} \mathrm{C}$ during which all systems are checked and initialized. data acquisition cycles will be determined as an offset fron this melting point. Data acquisition cycles are started after the succinonitrile is 
fully molten. The thermostat In addition, a check of the melting point of the sample (succinonitrile, a cubic test material) is conducted allowing the temperature sensors to be calibrated to the actual melting point. This is an essential step in the initialization process since all subsequent undercoolings set and measured during the temperature is then lowered to the desired undercooling while crystal growth is suppressed by an inhibit heater until thermal equilibrium is attained throughout the sample. The inhibit heater is then turned off and dendritic growth is allowed to occur. Photographs will be taken after each millimeter of dendritic growth. The sample is then reheated above the melting point until it becomes fully molten and the cycle is repeated at a new undercooling. A total of ten undercoolings, ranging from $0.1 \mathrm{~K}$ to $1.0 \mathrm{~K}$ in increments of $0.1 \mathrm{~K}$, will each be examined twice during the course of the STS mission.

\section{IDGE EXPERTMIENTAL APPARATUS}

The actual flight version of the experimental apparatus is still in the initial development stage at NASA Lewis Research Center and Rensselaer Polytechnic Institute. The isothermal dendrite growth experiment will be mounted on the Material Science Laboratory (MSL) in the shuttle cargo bay and will be essentially autonomous, except for a limited number of preprogrammed computer commands. The apparatus consists of four major subsystems: 1) a temperature controlled thermostatic bath, 2) a photographic data collection system, 3) a start detection system to initiate data collection and 4 ) a crystal growth chamber. The delicate laboratory growth chamber, Figure $6 a$, is being redesigned for the flight experiment. The current development of a growth chamber for flight aboard STS, Figure $6 \mathrm{~b}$, has focused on the following two aspects: A) examination of the effect of the design of the growth chamber on the crystal growth processes and the resultant dendrite orientation and $B$ ) identification of suitable construction materials which are chemically compatible with succinonitrile, such that any impurity-induced melting point changes do not exceed $10 \mathrm{mk}$. The present discussion will describe the dendrite orientation testing. A more detailed description of the other components and their performance requirements can be found elsewhere [15].

\section{DEADRITE ORIEATATIOA TESTS}

The purpose of these tests is to identify the growth chamber design most favorable for growth of an oriented single crystal, resulting in a single dendrite growing coaxially from the capillary tip (see Figure 6b). If this can be achieved, the photographic system can be aligned such that the focal planes would be coincident with the 


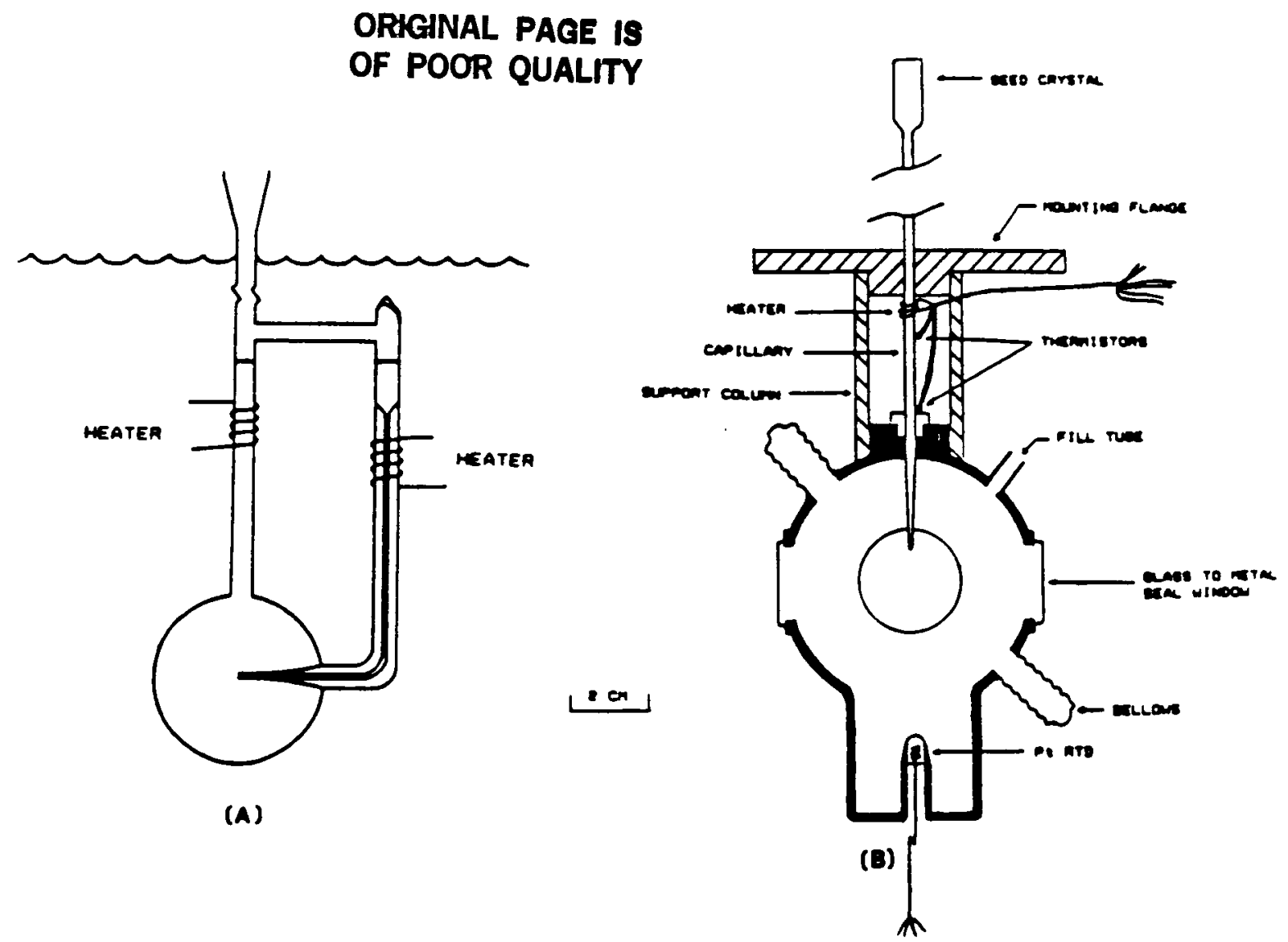

Figure 6 (a) The laboratory growth chamber used in ground based studies. (b) The current design of the flight growth chamber.

dendrite, providing clear sharp images of the dendrite during the entire course of its growth. The kinetic and morphological measurements can be measured directly from the negative with small, if any, corrections needed to obtain the true velocity and true shape of the dendrite. Two common techniques to obtain an oriented single crystal are either to furnish the growth chamber with a seed of the proper orientation or to provide a "crystal selector", such as a constriction or helix, in the capillary tube. It is uncertain, however, whether the seed crystal orientation can be maintained when the solid is propagated down the capillary.

Numerous laboratory growth chambers of varying designs have been constructed in order to examine the effect of design on the orientation of the resultant dendrite. The procedure used in conducting these tests is similar to the flight experiment procedure discussed previously. Crystallization is initiated when the inhibit heater is turned off and the solid grows as a thin film along the inside wall of the capillary tube. The advancing solid-liquid interface is not planar, but 
appears to be cellular at low undercoolings $(<0.5 \mathrm{~K})$ and dendritic at larger undercoolings $(>0.5 \mathrm{~K})$. The solid film emerges from the capillary tip and breaks down into dendrites which grow outward into the melt. The first series of tests examined the influence of the capillary tip material, and its size and shape on the orientation of the resultant dendrite. The results of these experiments indicate that these factors have little influence over the orientation of the emerging dendrite; however, size and shape of the tip do affect the manner and number of dendrites growing from the tip. In almost all cases a single crystal, the orientation of which varied randomly from test to test, emerged from the tip, even though a seed crystal was not used. This is clearly illustrated in Figure 7 where the observed growth angle with respect to vertical is plotted against the number of the observation. The mean value of growth angle for these observations is approximately $25^{\circ}$, which corresponds to the mean value expected for a completely random distribution. This behavior may be attributed to one of the following: 1 ) the starting solid was multicrystalline, but crystal selection occurred in the capillary prior to the emergence of the dendrite, 2) the starting solid was a single crystal but growth down the capillary was multicrystalline with

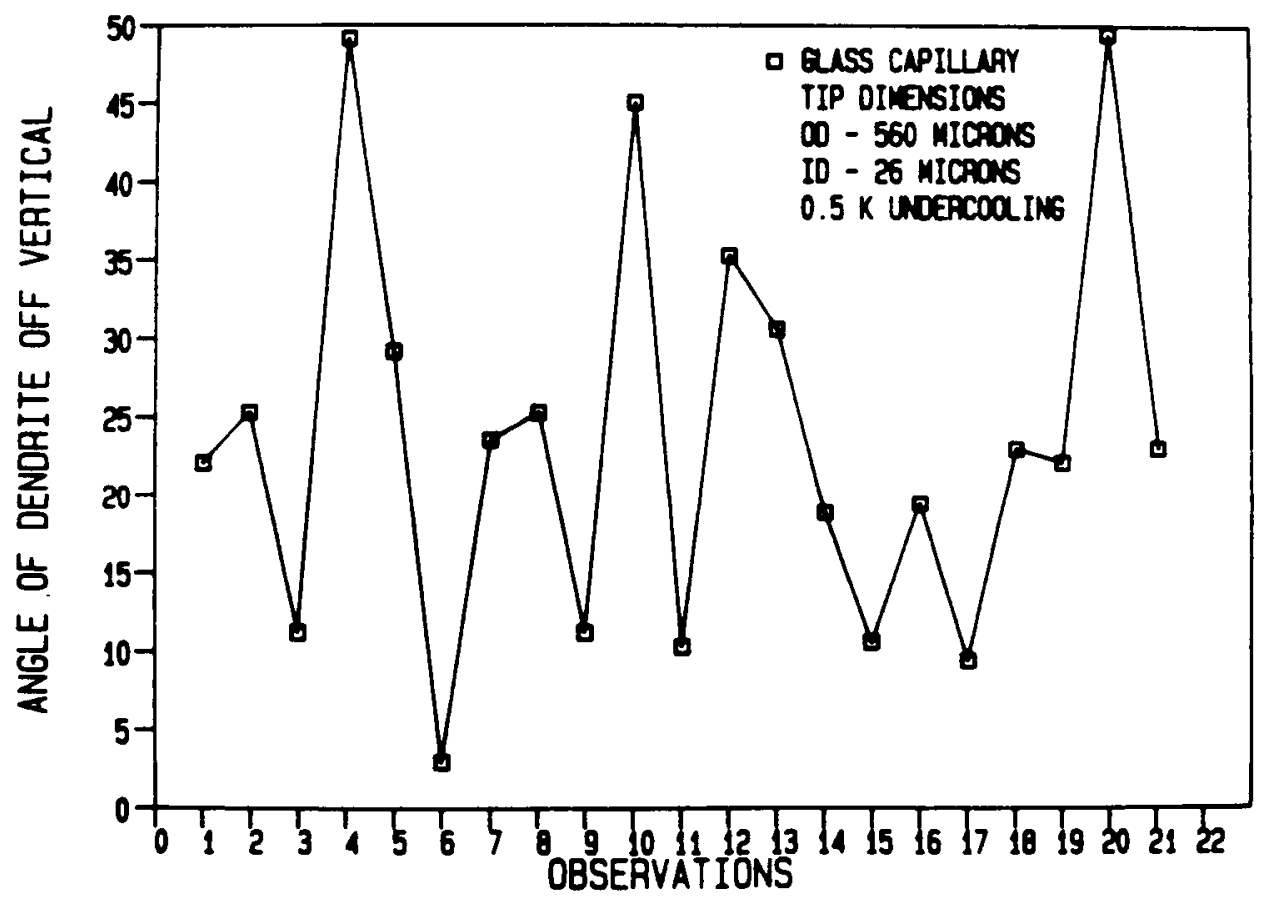

Figure 7 Observed dendrite growth angle with respect to the capillary axis as a function of the number of the test. 
crystal selection occurring in the capillary, or 3 ) the starting solid and growth down the capillary were single crystalline but the original single crystal was destroyed between tests and replaced inadvertently by a another single crystal of differing orientation. It is believed that the first and third possibilities could be easily resolved by seeding and careful procedural control to ensure the integrity of the seed crystal. If growth down the capillary is multicrystalline, however, it may be impossible to achieve an oriented dendrite without some mechanism to repeatedly select a crystal with the proper orientation. Furthermore, this issue raises the question of the need for a seed crystal of the proper orientation if, in fact, it is not possible to propagate that seed into a undercooled melt. Further testing will attempt to identify the necessary conditions, if any, where growth of a single crystal from a undercooled melt is possible.

\section{SUMAARY}

The IDGE is an active crystal growth experiment designed to test dendritic growth theory at low undercoolings where convection prohibits such studies at 1g. The experiment will be essentially autonomous, though limited in-flight interaction through a computer interface is planned. One of the key components of the apparatus will be a crystal growth chamber capable of achieving oriented single crystal dendritic growth. Recent work indicates that "seeding" the chamber with a crystal of the proper orientation will not, in and of itself, be sufficient to meet this requirement. Additional flight hardware and software required for the STS flight experiment are currently being developed at Nasa Lewis Research Center and at Rensselaer Polytechnic Institute.

\section{ACRTONLEDGERTI}

This work is being conducted with the support of the Materials Processing in Space Program, National

Aeronautics and Space Administration under NAG 3-333 with liaison through the Lewis Research Center.

\section{REFEREATCES}

1. M. E. Glicksman and S. C. Huang, Adv. Space Res., Vol 1, 25 (1981).

2. S. C. Huang and M. E. Glicksman, Acta Met, Vol 29,701 (1981).

3. M. E. Glicksman, R. J. Schaefer and J. D. Ayers, Met Trans A, Vol. 7A, 1747 (1976). 
4. G. P. Ivantsov, Dokl. Akad. Nauk, USSR, Vol 58, 567 $(1947)$.

5. J. S. Langer and H. Muller-Krumbhaar, Acta Met, Vol 26, 1681 (1978).

6. J. S. Langer and H. Muller-Krumbhaar, Acta Met, Vol 26,1689 (1978).

7. J. S. Langer and $H$. Muller-Krumbhaar, Acta Met, Vol 26,1697 (1978).

8. D. Kessler, J. Koplik and H. Levine, Phys. Rev., Vol. A30, 3161 (1984).

9. E. Ben-Jacob, N. Goldenfeld, G. Kotliar and

J. Langer, Phys. Rev. Lett., Vol. 53, 2110 (1984).

10. J. S. Langer, Phys. Rev., Vol. A33, 475 (1986)

11. D. Kessler, J. Koplik and H. Levine, Proceedings of NATO A.R.W., "Patterns, Defects and Microstructures in Non-Equilibrium Systems", (1986) To be published.

12. N. Goldenfeld, Proceedings of the NATO ASI, "Physiochemical Hydrodynamics: Interfacial Phenomena" (1986) To be published.

13. Final Report - Low Acceleration Characterization of Space Station Environment, Revision A, SP85-MSFL2928, Prepared by Advanced Programs Department, Space Programs Division, Teledyne Brown Engineering, Huntsville, Alabama (1985).

14. D. Langbein, "Allowable G-levels for Microgravity Payloads", To be published in ESA Journal.

15. M. E. Glicksman, E. Winsa, R. C. Hahn, T. A. Lograsso, E. R. Rubinstein and M. E. Selleck, "Solidification Under Microgravity Conditions Dendritic Growth", To be published in AAS Journal. 